\title{
Competencias de los egresados universitarios con prácticas realizadas en la suite comunicación
}

\section{University graduates'skills with internship in la suite communication}

\author{
Ana María Morales Rodríguez*, Francisco Manuel Morales Rodríguez**,... \\ *Universidad de Málaga **Universidad de Granada
}

\begin{abstract}
Resumen
El artículo analiza la experiencia práctica en la empresa, La Suite Comunicación, con el objetivo de definir las competencias que los egresados universitarios adquieren para su currículo laboral a través de la inserción en el mundo profesional. La situación laboral en la que está sumergida el país sumada al número de jóvenes con títulos que salen cada año de las universidades y la incompatibilidad que hay entre la formación recibida y la demanda de las empresas han creado un panorama para el recién graduado en el que las prácticas de empresa es una de las puertas al mundo laboral.
\end{abstract}

Palabras clave: graduado, universidad, prácticas, empresa.

\begin{abstract}
This paper analyzes the internship experience in the company La Suite Communication with the objective of defining the competences that the university graduates gain for their curriculum through the insertion in the professional world. The employment situation in which the country is submerged, with the number of young people with degrees that finish each year the university and the mismatch between the training received and the demand of the companies have created a scene for the recent graduate in which internships are one of the doors to the professional area.

Keywords: graduated, university, internship, company.
\end{abstract}

La situación laboral en la que esta sumergida el país sumada al número de jóvenes con títulos universitarios y de posgrado que salen cada año de las universidades y al desajuste que hay entre la formación recibida y la demanda de las empresas han creado un panorama para el recién graduado en el que las prácticas de empresa son la única puerta al mundo laboral. Como muchas otras empresas, La Suite Comunicación establece acuerdos de prácticas con varias universidades españolas a través de los cuales ayuda a los recién egresados a crear una primera toma de contacto con el mundo laboral. A través de esta pasantía los jóvenes adquirirán una serie de conocimientos y competencias que conectarán la experiencia universitaria con la experiencia laboral. Todo ello ayudará al "desarrollo constructivo de habilidades, conocimientos y actitudes que permitan a los futuros profesionales insertarse adecuadamente en la estructura laboral y adaptarse a los cambios sociales" (como se cita en Gil Cubillos, 2010),

Sin embargo, según las tareas y responsabilidades que se registren en el Plan de Prácticas diseñado por la empresa, han de elegirse una serie de competencias que exijan la realización de actividades vinculadas al área de estudio y profesional que cada graduado vaya a desempeñar en la empresa (Villa \& Poblete, 2011).

"Las competencias profesionales de los trabajadores se definen como un conjunto identificable y evaluable de conocimientos, aptitudes, valores y habilidades relacionadas entre sí, que permiten desempeños satisfactorios en situaciones reales de trabajo, según estándares utilizados en el área ocupacional" (Freire \& Salcines, 2010).

Por ello, La Suite Comunicación considera las prácticas profesionales como un método para evaluar y conocer el nivel de la formación académica en cuanto a cubrir las demandas del mercado profesional de la comunicación, no sólo a la hora de la productividad, sino ante lo que la sociedad espera de los profesionales del sector. A través de esta estancia en la empresa se puede analizar el primer acercamiento del estudiante con la experiencia profesional donde no sólo se mide la calidad del desempeño y la habilidad para responder ante las demandas, sino que se exigen ciertas competencias sociales que permitan al estudiante adaptarse al ambiente de trabajo (Durán-Aponte \& Durán-García, 2012).

\section{Método}

El objetivo básico de este estudio es definir y analizar qué competencias desprenden los graduados universitarios durante su periodo de prácticas en La Suite Comunicación, a través de las cuales se persigue obtener información fiable de los puntos fuertes de los recién egresados para entrar al mundo laboral.

La Suite Comunicación diseñó un sistema de evaluación para dicho proceso en el cual la empresa pudiera conocer la experiencia del estudiante durante el periodo de prácticas y valorar su comportamiento y conocimiento adquirido durante dicho proceso.

Para que la experiencia de las prácticas en empresa se convierta en un verdadero proceso de aprendizaje debe contar, al menos, con tres características: 
- a) integrar experiencias bien planificadas y coherentes con las competencias a desarrollar;

- b) favorecer la reflexión sobre la experiencia;

- c) facilitar la integración de la experiencia a través de la autoevaluación, el análisis de las consecuencias y la potenciación de la transferencia a otras situaciones. (como se cita en Talavera \& Pérez-González, 2007).

Otro de los aspectos fundamentales para la experiencia de prácticas es la asignación de un tutor dentro de la empresa. En este caso, la tutora de prácticas asignada para los recién titulados que acuden a La Suite Comunicación es la ejecutiva de cuentas y directiva de proyectos, Carmen Murillo Lechuga. A través de la figura de la tutora, desde la empresa, se pretende dar orientación, ayuda y atención personalizada durante el tiempo que dure la pasantía. A través de la experiencia de los graduados con la tutora, en primer plano, y con el resto de compañeros, en segunda instancia, se establecerán las competencias a analizar y evaluar tras finalizar el periodo de prácticas.

\section{Participantes}

Universitarios recién graduados de estudios de grado o postgrado que hayan completado su periodo de prácticas en La Suite Comunicación.

\section{Instrumentos o materiales}

Para la correcta evaluación del egresado se procedió al diseño de un Plan de Prácticas, que se aplicará a cada uno de los estudiantes que realice su periodo de pasantía en La Suite Comunicación. Dicho plan está compuesto por los datos del estudiante o recién egresado, datos de la empresa en la que se realiza el periodo de prácticas, datos del tutor asignado por la empresa, actividades desarrolladas y duración de las prácticas, capacidades o competencias, apartado que se analiza en dicho artículo, y, por último, las observaciones.

\section{Procedimiento}

A la hora de incorporar las competencias evaluadas, se hace distinción entre competencias específicas y competencias genéricas que exijan la realización de las actividades desempeñadas por el recién graduado, las cuales siempre corresponden al perfil académico-profesional de la titulación para la cual se ha formado el estudiante (Villa \& Poblete, 2011).

\section{Resultados}

Si se tienen en cuenta todos los aspectos anteriores, junto a la experiencia del tutor con los estudiantes, los resultados de las tareas desempeñadas, la relación con el equipo profesional de La Suite Comunicación y las habilidades básicas que se necesitan en una empresa del sector de la comunicación, se establecen una serie de competencias generales a evaluar en todos los perfiles:

- Administración de trabajos o tareas.

- Capacidad de aprendizaje.

- Creatividad.

- Facilidad de adaptación.

- Habilidad de comunicación oral.

- Habilidad de comunicación escrita.
- Iniciativa.

- Implicación personal.

- Motivación.

- Puntualidad.

- Receptividad a las críticas.

- Relación con el entorno laboral.

- Sentido de la responsabilidad.

- Trabajo en equipo.

Los alumnos, los cuales han superado estas competencias generales, se someten a la evaluación de estas específicas:

- Capacidad técnica vinculada al sector de la comunicación: para un informe favorable durante el desarrollo de las prácticas, el graduado deberá de estar familiarizado o conocer todos los programas informáticos y aplicaciones técnicas, o similares, necesarios para el correcto desarrollo de su trabajo en el sector de la comunicación, marketing, relaciones públicas o publicidad, diseño y comercio.

- Poseer, comprender y saber aplicar conceptos y técnicas fundamentales y avanzadas de la investigación social para su aplicación a proyectos del ámbito empresarial de la comunicación corporativa.

- Capacidad para ampliar los conocimientos adquiridos durante el periodo universitario y en la relación directa al mundo empresarial.

- Desarrollar en la empresa y de manera efectiva los conocimientos adquiridos durante el periodo de formación.

A través del análisis de este documento, la empresa ha podido comprobar que el egresado cumplía con las competencias que La Suite Comunicación exige a sus empleados para, en caso de demanda laboral, tener en cuenta dichas aptitudes y actitudes a la hora de seleccionar a un futuro empleado.

\section{Discusión}

Las prácticas profesionales en universitarios graduados para que se desenvuelvan en el ámbito laboral en el que fueron formados es, sobre todo, una gran oportunidad para aquellos que quieren acceder al mercado laboral sin tener experiencia previa.

A partir de la evaluación personalizada de dichas prácticas se puede estudiar el grado de profesionalización con la que los egresados acuden al ámbito laboral, además de la capacidad de estos para desempeñar tareas propias del sector en el que se han formado, enfocadas al beneficio empresarial.

El grado de exigencia de las competencias aplicadas a la evaluación de los recién graduados son superiores a las adquiridas en la etapa universitaria, lo que demuestra que los estudiantes experimentan un aumento de su nivel de formación en el sector profesional al que están enfocados. A través de las competencias generales analizadas por La Suite se concluye que aquellas asociadas al compromiso ético, la responsabilidad, el trabajo en equipo y la habilidad personal son las más demandadas y mejores evaluadas.

La orientación y tutoría por parte de la empresa es un aspecto esencial para que el graduado desarrolle de 
forma óptima las tareas durante su pasantía y por ende, finalice de manera positiva su primera relación con el mundo laboral.

Para La Suite Comunicación, aquellos graduados que hayan realizado prácticas durante el periodo de formación, o posteriormente, tienen mejores aptitudes y más posibilidades para acceder a un puesto de trabajo.

\section{Referencias}

Cassany, D., \& Ferrero, C. L. (2010). Capítulo 12. De la Universidad al mundo laboral: Continuidad y contraste entre las prácticas letradas académicas y profesionales. Alfabetización académica y profesional en el siglo XXI: leer y escribir desde las disciplinas. Barcelona: Planeta Ariel, 347-374. http://s3.amazonaws.com/academia.edu.documents/44 629618/Cassany_y_Lopez_Ferrero_septiembre_2009 _revisado_PUB.pdf?AWSAccessKeyId=AKIAIWO WYYGZ2Y53UL3A\&Expires $=1499096789 \&$ Signatu $\mathrm{re}=\mathrm{Ym}$ 9XvsofURR5LKEKoehCc2KEZ\%2Fw\%3D\&r esponse-content-disposition=inline $\% 3 \mathrm{~B} \% 20$ filename \%3DDe_la_Universidad_al_mundo_laboral_Conti.pd $\mathrm{f}$

Castillo, P. V., Venegas, C. B., Leiva, Y. F., Bennett, S. O., Ortiz, E. P., \& de Souza Neto, B. (2016). Una innovación pedagógica para la formación de universitarios emprendedores. Revista da FAE, 11(2). https://revistafae.fae.edu/revistafae/article/view/331

Cruz, N. M., Barahona, J. J. H., \& Escudero, A. I. R. (2005). Análisis de la formación y la experiencia laboral como determinantes del espíritu emprendedor de los estudiantes universitarios. RAE: Revista Asturiana de Economía, (34), 131-145. https://dialnet.unirioja.es/servlet/articulo?codigo $=223$ 5356

Durán-Aponte, E., \& Durán-García, M. (2012). Competencias sociales y las prácticas profesionales. Vivencias y demandas para la formación universitaria actual. Cultura y Educación, 24(1), 61-76. http://www.tandfonline.com/doi/abs/10.1174/1135640 12799740777

Freire Seoane, M. J., \& Salcines Cristal, J. V. (2010). Análisis de las competencias profesionales de los titulados universitarios españoles: La visión de los egresados. Perfiles educativos, 32(130), 103-120. http://www.scielo.org.mx/scielo.php?pid=S0185-2698 $2010000400007 \&$ script=sci_arttext\&tlng=pt

Gil Cubillos, C. (2010). Comunicadores corporativos: desafíos de una formación profesional por competencias en la era global. Cuadernos del Centro de Estudios en Diseño y Comunicación. Ensayos, (33), 49-59.

http://www.scielo.org.ar/scielo.php?script=sci_arttext \&pid=S1853-35232010000300005

Martín, R. M. (2003). La inserción laboral de los universitarios a través de las prácticas en empresas. Reis, 229-254. http://www.jstor.org/stable/40184457?seq=1\#page_sc an_tab_contents
Sánchez-Olavarría, C. (2014). Los egresados de comunicación y el mercado laboral: un estudio de trayectorias profesionales. Revista iberoamericana de educación superior, 5(13), 40-54. http://www.scielo.org.mx/scielo.php?pid=S2007-2872 2014000200003\&script=sci_arttext\&tlng=pt

Talavera, E. R., \& Pérez-González, J. C. (2007). Formación en competencias socioemocionales a través de las prácticas en empresas. Revista Europea de Formación Profesional, (40), 92-112. http://portal.uned.es/pls/portal/docs/PAGE/UNED_M AIN/LAUNIVERSIDAD/UBICACIONES/03/DOCE NTE/JUAN_CARLOS_PEREZ_GONZALEZ/REPE TTO $\% 20 \% 26 \% 20 \mathrm{P} \% \mathrm{C} 3 \% 89 \mathrm{REZ}-\mathrm{GONZ} \% \mathrm{C} 3 \% 81 \mathrm{~L}$ EZ,\%202007_EJVT,\%2040_ESPA\%C3\%91OL_0.P $\mathrm{DF}$

Villa Sánchez, A., \& Poblete Ruiz, M. (2011). Practicum y evaluación de competencias. http://www.redalyc.org/pdf/567/56780203.pdf 